\title{
Perfil epidemiológico e análise espacial da hanseníase na área de atuação da Estratégia Saúde da Família
}

\author{
Epidemiological profile and spatial analysis of leprosy in the action area of the Family Health
} Strategy

Perfil epidemiológico y análisis espacial de la lepra en la área de actuación de la Estrategia de Salud Familiar

\author{
Kaio Pantoja de Lima \\ ORCID: https://orcid.org/0000-0003-1068-2554 \\ Universidade Federal do Pará, Brasil \\ E-mail: kaioplima@gmail.com \\ Emanuele Rocha da Silva \\ ORCID: https://orcid.org/ 0000-0002-9109-4158 \\ Universidade Federal do Pará, Brasil \\ E-mail: emanueleersilva@gmail.com \\ Francisco Cezar Aquino de Moraes \\ ORCID: https://orcid.org/0000-0003-0623-8135 \\ Universidade Federal do Pará, Brasil \\ E-mail: francisco.cezar2205@gmail.com \\ Izaura Maria Vieira Cayres Vallinoto \\ ORCID: https://orcid.org/0000-0003-1408-8384 \\ Universidade Federal do Pará, Brasil \\ E-mail: ivallinoto@ufpa.br \\ Waltair Maria Martins Pereira \\ ORCID: https://orcid.org/0000-0002-7383-0318 \\ Universidade Federal do Pará, Brasil \\ E-mail: vweapaz@gmail.com
}

\begin{abstract}
Resumo
Objetivo: Descrever o perfil epidemiológico, a distribuição espacial dos casos de hanseníase e a qualidade do serviço de uma Estratégia Saúde da Família (ESF) localizada no município de Belém, estado do Pará, de 2008 a 2015. Método: Estudo quantitativo descritivo transversal, a partir de fichas arquivadas no Sistema de Informação de Agravos de Notificação. A população estudada foi constituída pelos casos de hanseníase residentes do município de Belém, notificados por uma ESF. Através do método de Kernel, foi criado o mapa temático com a densidade de casos. Os dados trabalhados foram apresentados em forma de tabelas e gráficos elaborados no Microsoft Office Excel 2013 e mapas temáticos no software Qgis, 2017. Resultados: Dos 14 casos registrados no período, a maior ocorrência da doença foi no gênero masculino $(71,43 \%)$, cor da pele parda $(42,86 \%)$, com baixa escolaridade $(57,14 \%)$ e com predomínio da faixa etária de 20 a 39 anos (43\%). O primeiro e o último anos do estudo apresentaram a menor taxa de detecção da doença $(0,4 / 1.000$ hab.), enquanto em 2013 e 2014 foi registrado o valor mais expressivo da série (1,6/1.000 hab.). O método de Kernel evidenciou diferentes densidades de interesse na superfície da área adstrita à ESF, com atendimento de casos que residiram fora desta $(42,86 \%)$. Conclusão: A persistência da endemia e a alta proporcionalidade de atendimentos a casos advindos de fora da área adstrita à ESF demonstram a necessidade de expansão da sua cobertura populacional e auxiliam a um planejamento direcionado à população com maior risco de adoecimento.
\end{abstract}

Palavras-chave: Hanseníase; Análise espacial; Estratégia saúde da família.

\section{Abstract}

Objective: To describe the epidemiological profile, the spatial distribution of leprosy cases and the service quality of a Family Health Strategy (FHS) located in Belém, Pará state, from 2008 to 2015. Methodology: Cross-sectional quantitative study, based on Information System and Notifiable Diseases files. The studied population consisted of leprosy cases resident in Belém, notified by an FHS. Through the Kernel method, the thematic map with the density of cases was created. The data were presented in the form of tables and graphs in Microsoft Office Excel 2013 and thematic maps in the software Qgis, 2017. Results: Of the 14 cases in the period, the highest occurrence of the disease was in the male gender (71.43\%), brown skin color $(42.86 \%)$, with low education $(57.14 \%)$ and with a predominance of the age group of 20 to 39 years (43\%). The first and last years of the study showed the lowest rate of detection of 
the disease (0.4 / 1,000 inhab.), While in 2013 and 2014 the most expressive value of the series was recorded (1.6 / 1,000 inhab.). The Kernel method showed different densities of interest on the surface of the area assigned to the ESF, with care for cases that resided outside it (42.86\%). Conclusion: The persistence of the endemic disease and the high proportionality of attendance to leprosy cases outside the area assigned to the FHS demonstrate the importance of this service and allow the directing of planning to the population at greatest risk of illness.

Keywords: Leprosy; Spatial analysis; Family health strategy.

\section{Resumen}

Objetivo: Describir el perfil epidemiológico, la distribución espacial de los casos de lepra y la calidad de servicio de una Estrategia de Salud de la Familia (ESF) en Belém, Pará, de 2008 a 2015. Metodologia: Estudio cuantitativo transversal, basado en archivos del Sistema de Información y Enfermedades Notificables. La población fue constituida por casos de lepra residentes en el municipio de Belém, notificados por una ESF. Mediante el método Kernel se creó el mapa temático con la densidad de casos. Los datos trabajados se presentaron en tablas y gráficos elaborados en Microsoft Office Excel 2013 y mapas temáticos en el software Qgis, 2017. Resultados: De los 14 casos registrados en el período, la mayor ocurrencia de la enfermedad fue en el sexo masculino (71,43\%), color de piel morena $(42,86 \%)$, con baja escolaridad $(57,14 \%)$ y con predominio del grupo de edad de 20 años. a 39 años (43\%). El primer y último año del estudio mostró menor tasa de detección (0,4 / 1.000 hab.), mientras que en 2013 y 2014 se registró el valor más expresivo (1,6 / 1.000 hab.). El método Kernel mostró diferentes densidades en la superficie del área asignada a la ESF, con atención para los casos que residían fuera de ella (42,86\%). Conclusión: La persistencia de la enfermedad y la alta proporcionalidad de atención a casos provenientes de fuera del área asignada a la ESF demuestran la necesidad de ampliar su cobertura poblacional y planificación dirigida a la población con mayor riesgo de enfermedad.

Palabras clave: Lepra; Analisis espacial; Estrategia de salud familiar.

\section{Introdução}

A hanseníase é uma doença infectocontagiosa crônica, causada pelo Mycobacterium leprae, um parasita intracelular obrigatório que possui tropismo pela pele e pelos nervos periféricos, manifestando-se, principalmente, por meio de sinais e sintomas dermatoneurológicos (Benjak, et al., 2018). Seu curso crônico pode ser interrompido por períodos de piora clínica das lesões, representados pelos estados reacionais (BRASIL, 2014). A prevalência da hanseníase a nível mundial diminuiu de $>5$ milhões de casos em meados da década de 1980, para < 200.000 em 2015, após a introdução da poliquimioterapia (PQT) no tratamento medicamentoso (WHO, 2015).

O diagnóstico precoce e o tratamento completo com a PQT continuam a figurar entre as principais estratégias para reduzir a carga da hanseníase No primeiro trimestre de 2015, o número de casos registrados no mundo chegou a 175.554, determinando prevalência de 0,31 por 10.000 habitantes, demonstrando um discreto decréscimo em relação ao ano anterior cuja prevalencia foi de 0,32 (WHO, 2015). O sudeste asiático apresentou o maior número de casos registrados (119.478), seguido das Américas (29.967) e África (19.968), com a prevalência de 0,63; 0,33 e 0,26 por 10.000 habitantes, respectivamente. O Brasil, a Índia e a Indonésia são responsáveis por $81 \%$ dos casos novos de hanseníase no mundo todo (WHO, 2015). O Brasil, no ano de 2014, apresentou 25.738 casos de hanseníase em registro ativo, com taxa de prevalência geral de 1,27 por 10 mil habitantes; prevalência em menores de 15 anos de 4,88 por 10 mil menores de 15 anos; taxa geral de detecção de casos novos de 15,32 por 100 mil habitantes e proporção de pacientes multibacilares de 65,91\% (Brasil, 2014).

Os princípios do Sistema Único de Saúde (SUS) são incorporados pela Estratégia Saúde da Família (ESF) através da execução da Atenção Primária em Saúde (APS). A ESF representa a porta de entrada da Rede de Atenção a Saúde (RAS) e, portanto, realiza o primeiro contato com os indivíduos da comunidade, realiza a longitudinalidade, a abrangência do cuidado e a coordenação e orientação à família e à comunidade (Brasil, 2017). A implantação de ações de controle da hanseníase em todas as unidades de saúde da RAS se apresenta como uma das soluções para o alcance da meta de controle da doença. Com custos reduzidos, devido não exigir procedimentos de alta complexidade, os medicamentos e a vacina BCG serem fornecidos pelo Ministério da Saúde, sendo de responsabilidade dos municípios a condução da gerência do controle da hanseníase com o acompanhamento da gestão dos Estados (Morosini, Fonseca \& Lima, 2018; Brasil, 2017). 
O objetivo deste estudo foi descrever o perfil epidemiológico, a distribuição espacial dos casos de hanseníase e a qualidade do serviço de uma ESF, localizada no município de Belém, no período de 2008 a 2015.

\section{Metodologia}

Pesquisa quantitativa com desenho de estudo descritivo transversal, realizada no município de Belém, estado do Pará (Pereira, et al., 2018). A população do estudo consiste nos casos de hanseníase notificados em uma ESF, no período de 2008 a 2015. Foram incluídos indivíduos de ambos os gêneros, de todas as faixas etárias, com ficha de notificação e investigação de hanseníase cadastrada no Sistema de Informação de Agravos de Notificação (SINAN), com endereço constando o logradouro, número e bairro, e assinada por profissional lotado na ESF, atendidos no período do estudo. Foram excluídos os casos que tiveram mudança de diagnóstico ou preenchimento incompleto da ficha. As variáveis de interesse trabalhadas foram idade, sexo, raça/cor da pele, escolaridade, bairro de residência, classificação clínicas da doença; tipo de entrada no SINAN e o número de contatos intradomiciliares registrados e examinados.

No estudo, foram consideradas as formas clínicas indeterminada, tuberculóide, dimorfo e Virchowiana. Os indicadores operacionais de monitoramento e avaliação da hanseníase, que medem a força da morbidade, a magnitude e o perfil epidemiológico, além da qualidade das ações e serviços de saúde. Os indicadores foram calculados seguindo as orientações do manual de Diretrizes para Vigilância, Atenção e Eliminação da Hanseníase como Problema de Saúde Pública (Brasil, 2016). A partir dos dados contidas nas fichas de investigação notificação de casos da doença, sobre logradouro e número da residência, os casos foram identificados no banco de dados do SINAN para confirmação das notificações.

Através do Google Maps e do Google Street View, foram localizados os endereços, marcadas as coordenadas geográficas de longitude e de latitude e os endereços de cada caso foi georreferenciado. Foi constituído o banco de dados geográficos (BDGEO) que proporcionou a elaboração de mapas temáticos com a localização geográfica dos endereços dos casos notificados de hanseníase e a localização da ESF em questão, utilizando o shape file do município de Belém, dos Bairros Terra Firme e Universitário e das ruas de ambos os bairros. A partir de polígonos, foi criado o shape file das 21 micro-áreas de atuação da equipe da ESF, em particular dos Agentes Comunitários de Saúde (ACS). Os dados trabalhados foram apresentados em forma de tabelas, gráficos e mapas. Para a construção das tabelas e gráficos foi utilizado o software Microsoft Office Excel 2013. A densidade de casos na área adstrita à ESF foi identificada pelo método de Kernel e a análise espacial da evolução do número de casos foi realizada pela expressão visual através de mapa coroplético.

O estudo foi realizado atendendo as determinações da Resolução $\mathrm{n}^{\mathrm{O}} 466$ de 12 de dezembro de 2012, do Conselho Nacional de Saúde (CNS) que estabelece diretrizes e normas regulamentadores de pesquisas envolvendo seres humanos. Foi aprovado pelo Comitê de Ética e Pesquisa (CEP) do Instituto de Ciências da Saúde (ICS) da UFPA, com Certificado de Apresentação para Apreciação (CAAE) n 33158219.5.0000.0018; Parecer nº 677.575, de 30 de janeiro de 2015.

\section{Resultados}

A ESF notificou 14 casos de hanseníase, no período do estudo. A maior frequência da doença ocorreu no gênero masculino $(71,43 \%)$, em indivíduos de cor de pele parda $(42,86 \%)$ e pacientes com ensino fundamental incompleto $(57,14 \%)$ (Tabela 1). 
Tabela 1 - Casos novos de hanseníase, segundo gênero, escolaridade, e cor da pele. Estratégia Saúde da Família, Belém, estado do Pará, 2008 a 2015 (N=14).

\section{Características dos pacientes}

(N)

$\%$

\section{Gênero}

\section{Masculino \\ Feminino}

Total

\section{Cor da Pele}

Branca

Preta

Parda

Total
10

4

14

3

5

6

14
1,43

28,57

100,00

21,43

35,71

42,86

100,00

\section{Escolaridade}

Analfabeto

Ensino Fundamental Incompleto

Ensino Médio Completo

Ignorado

Sem Informação

Total

Fonte: SINAN (2017).

Foi alta a proporção de casos notificados em que não constam informações sobre a ocupação (35,72\%), correspondendo à maioria da população estudada. A maior proporcionalidade de ocupação foi a de estudantes $(21,44 \%)$.

Em relação à faixa etária, a população adulta prevaleceu, com predomínio dos indivíduos com idade entre 20 e 39 (43\%). As menores proporções corresponderam às faixas etárias de 10 a 14 anos e 15 a 19 anos, ambas com 7\%, demonstrando percentual baixo de casos notificados em crianças e em adolescentes (Figura 1).

Figura 1 - Proporção de casos novos de Hanseníase, por faixa etária. Estratégia Saúde da Família, Belém, estado do Pará, 2008 a $2015(\mathrm{~N}=14)$.

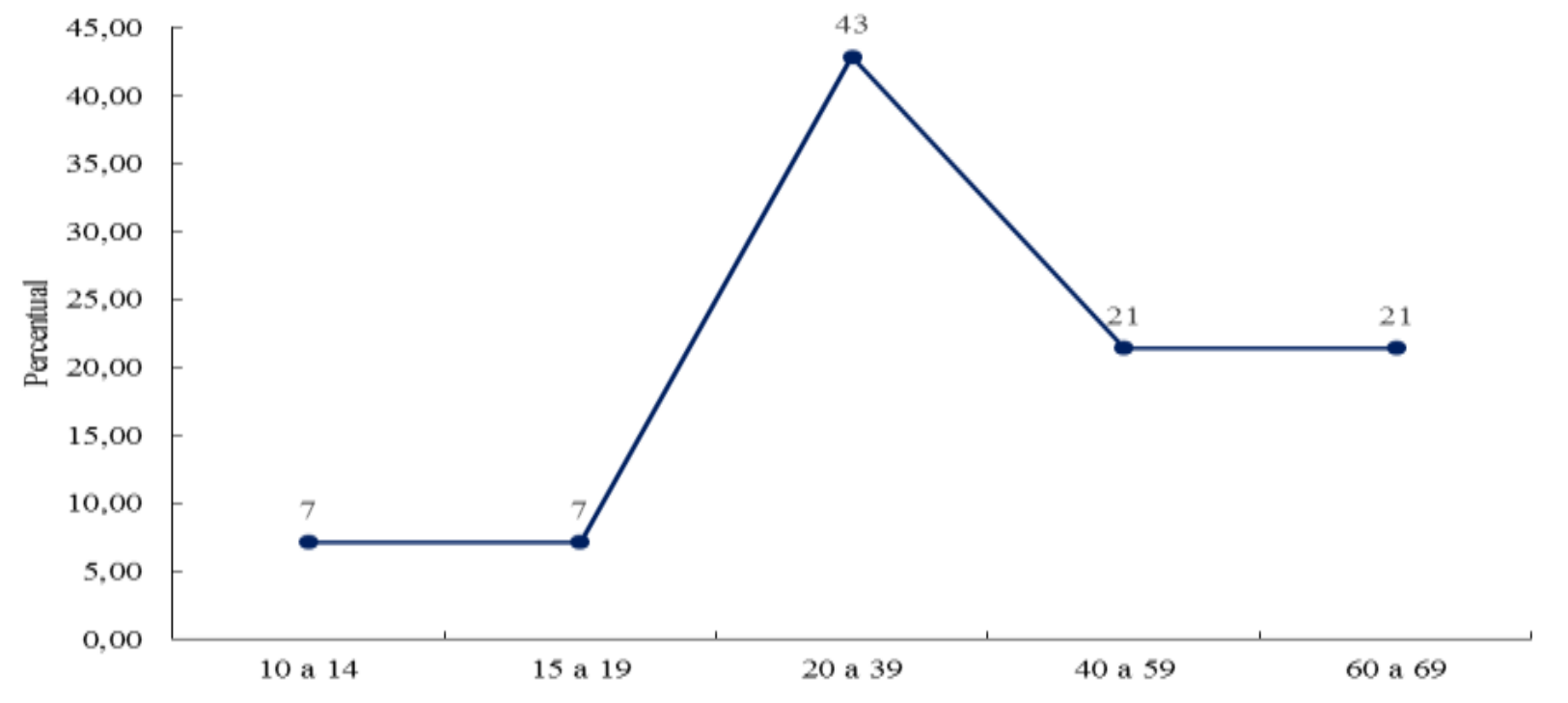


As formas clínicas apresentadas pelos pacientes no período estudado estão dispostas na Figura 2, onde pode ser identificada a predominância da detecção de formas clínicas já polarizadas, formas dimorfa, tuberculóide e Virchowiana.

Figura 2 - Hanseníase Por Forma Clínica. Estratégia Saúde da Família, Belém, estado do Pará, 2008 a 2015 (N=14).

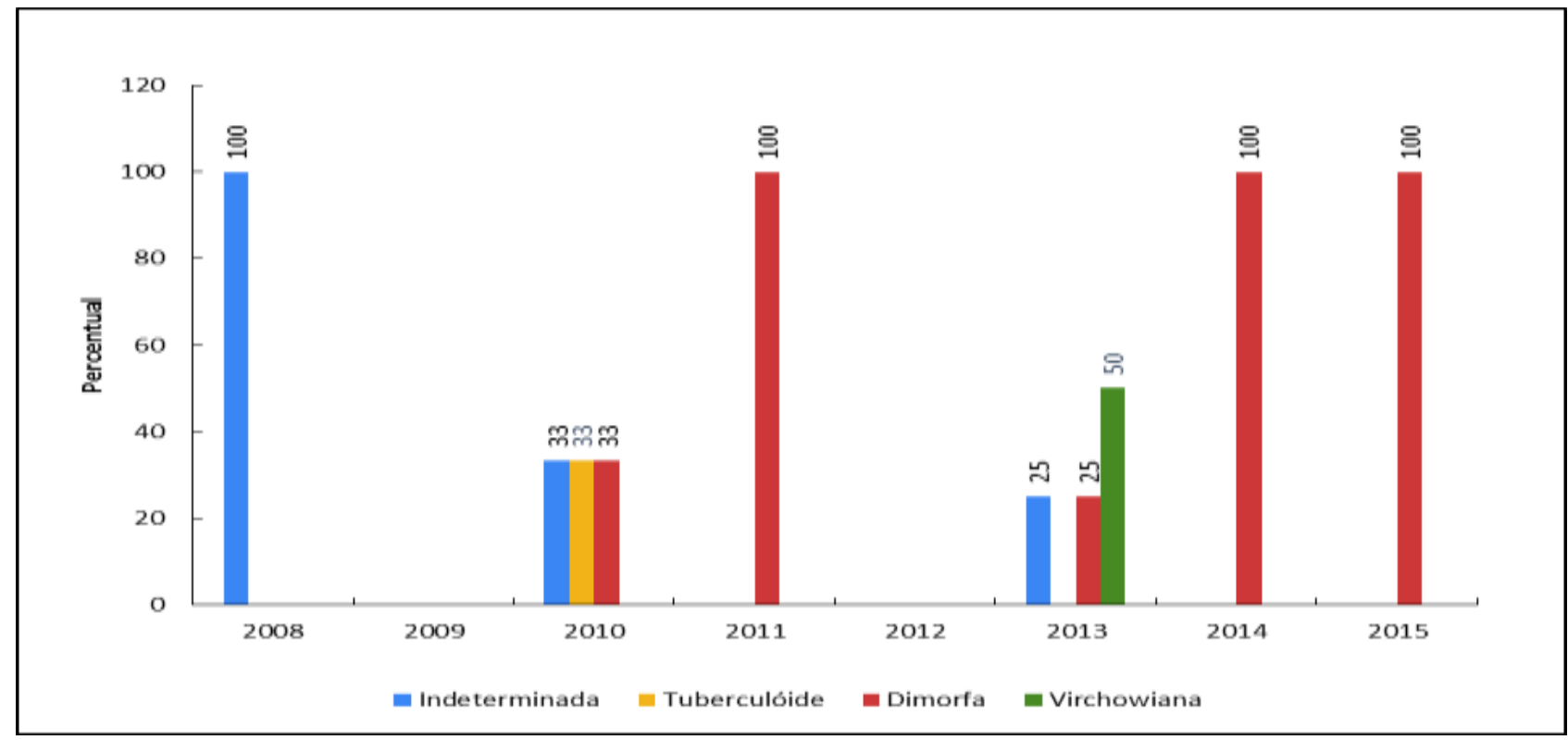

Fonte: SINAN (2017).

As taxas de detecção de hanseníase obtidas na área de atuação da ESF tiveram maior valor nos anos de 2013 e 2014 (1,60/1.000 hab.), sendo que o último ano do estudo foi um dos três com a menor taxa de detecção (0,4/1.000 hab) (Figura 3).

Figura 3 - Taxa de Detecção de Hanseníase, por 1.000 habitantes. Estratégia Saúde da Família, Belém, estado do Pará, 2008 a $2015(\mathrm{~N}=14)$.

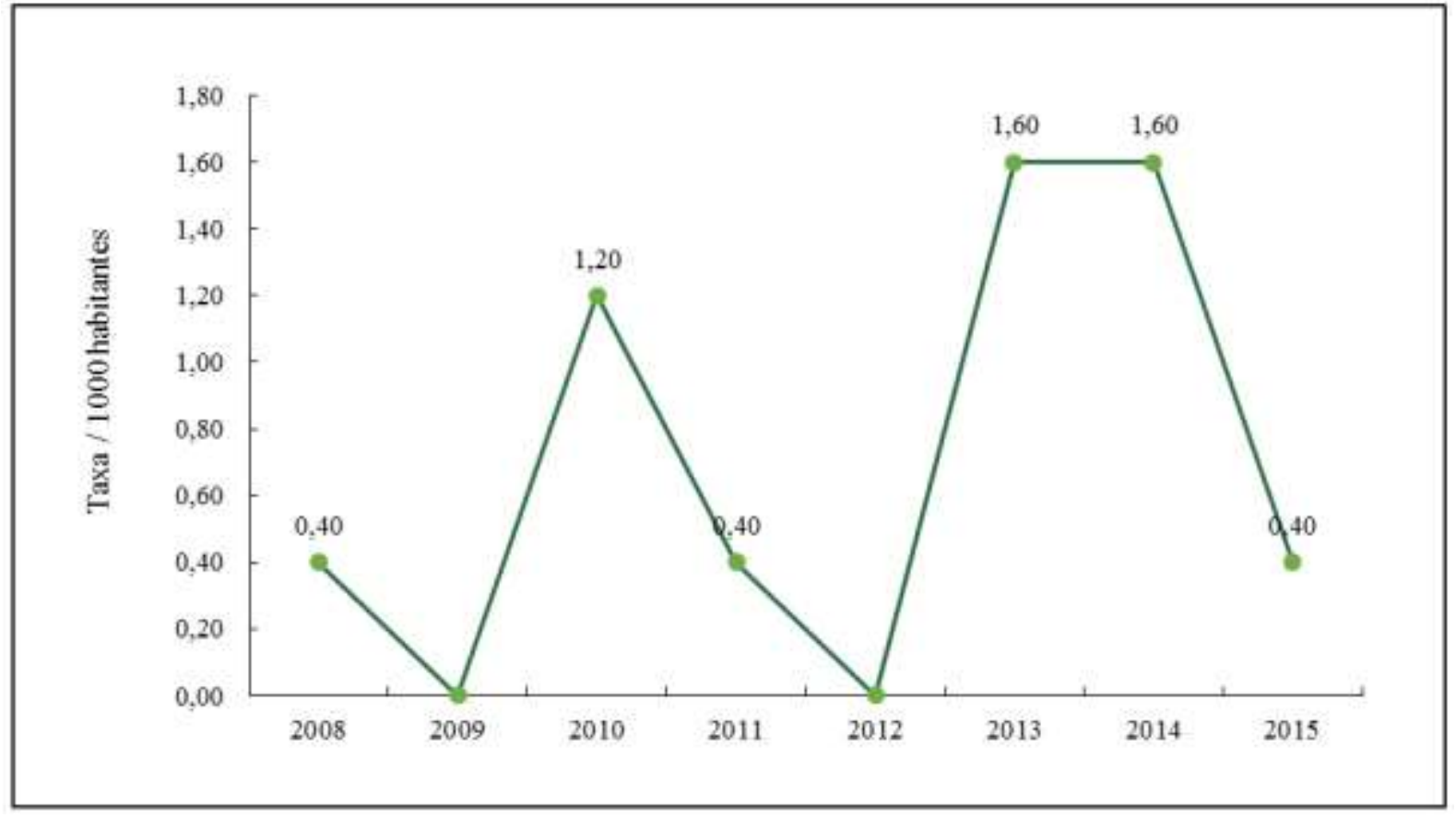

Fonte: SINAN (2017). 
Research, Society and Development, v. 10, n. 4, e58110414375, 2021

(CC BY 4.0) | ISSN 2525-3409 | DOI: http://dx.doi.org/10.33448/rsd-v10i4.14375

A área adstrita da ESF estudada é composta por 21 microáreas devidamente identificadas e delimitadas para o trabalho da equipe multiprofissional. Dos casos notificados, 42,86\% estavam localizados fora da área adstrita à ESF e 35,71\% localizavam-se no território bem próximo à ESF. Dos casos residentes na área adstrita, as maiores proporcionalidades foram de residentes nas microáreas A2 e A18, com 14,29\% cada uma delas. Em 15 microáreas, não foi observada residência de casos de hanseníase (Figura 4).

Figura 4 - Localização geográfica dos casos de hanseníase e da Estratégia Saúde da Família, Belém, estado do Pará, 2008 a 2015.

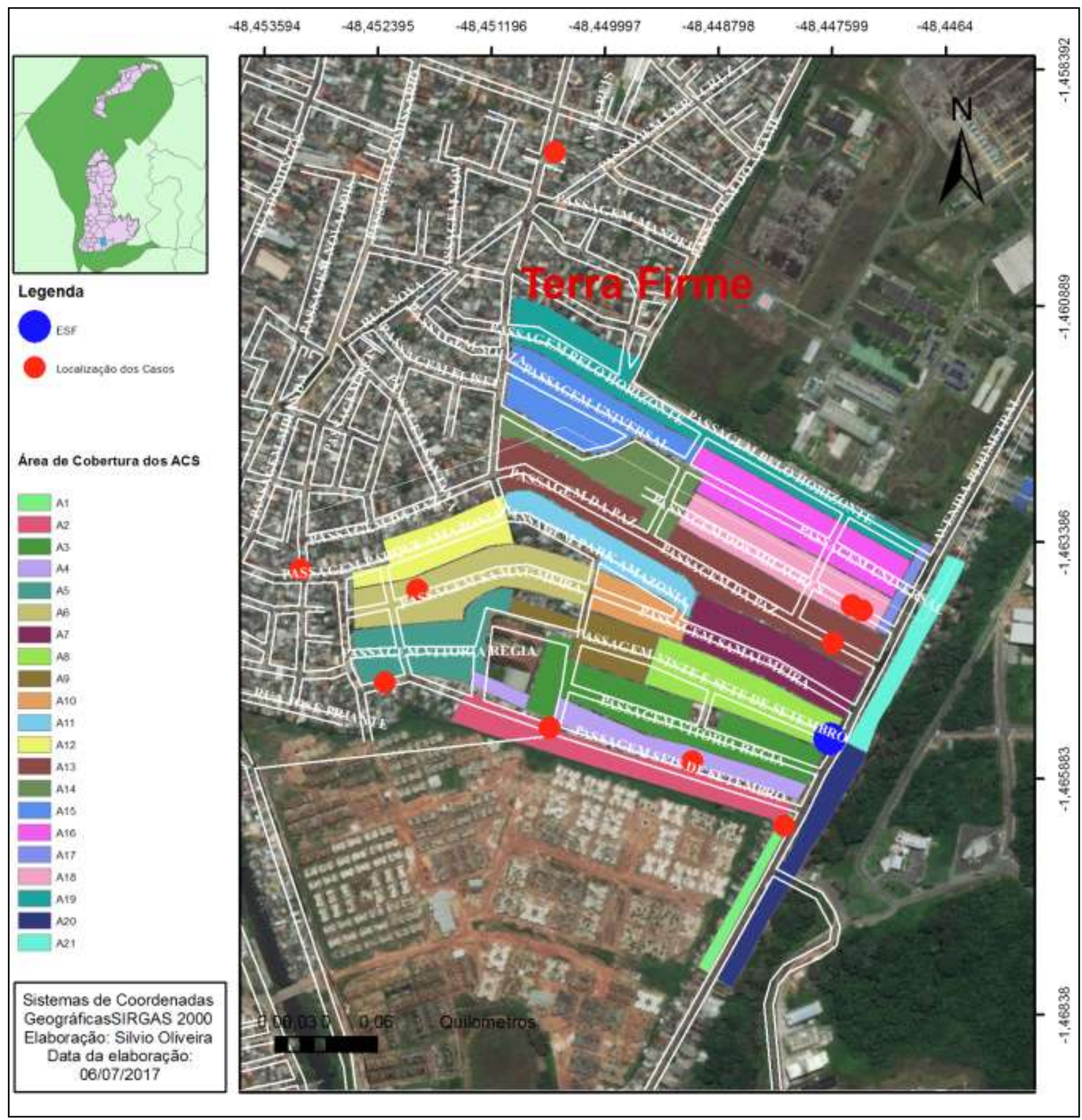

Fonte: Autores do trabalho (2017); Google maps (2017); Qgis (2017). 
Research, Society and Development, v. 10, n. 4, e58110414375, 2021

(CC BY 4.0) | ISSN 2525-3409 | DOI: http://dx.doi.org/10.33448/rsd-v10i4.14375

A maior densidade de casos notificados de hanseníase na área adstrita à ESF se fez presente nas microáreas A13 e A18 (9,52\%). Essas microáreas são contíguas e estão localizadas bem próximas à ESF (Figura 5).

Figura 5 - Densidade de casos de hanseníase na área adstrita da Estratégia Saúde da Família, Belém, estado do Pará, 2008 a 2015, $(\mathrm{N}=14)$.

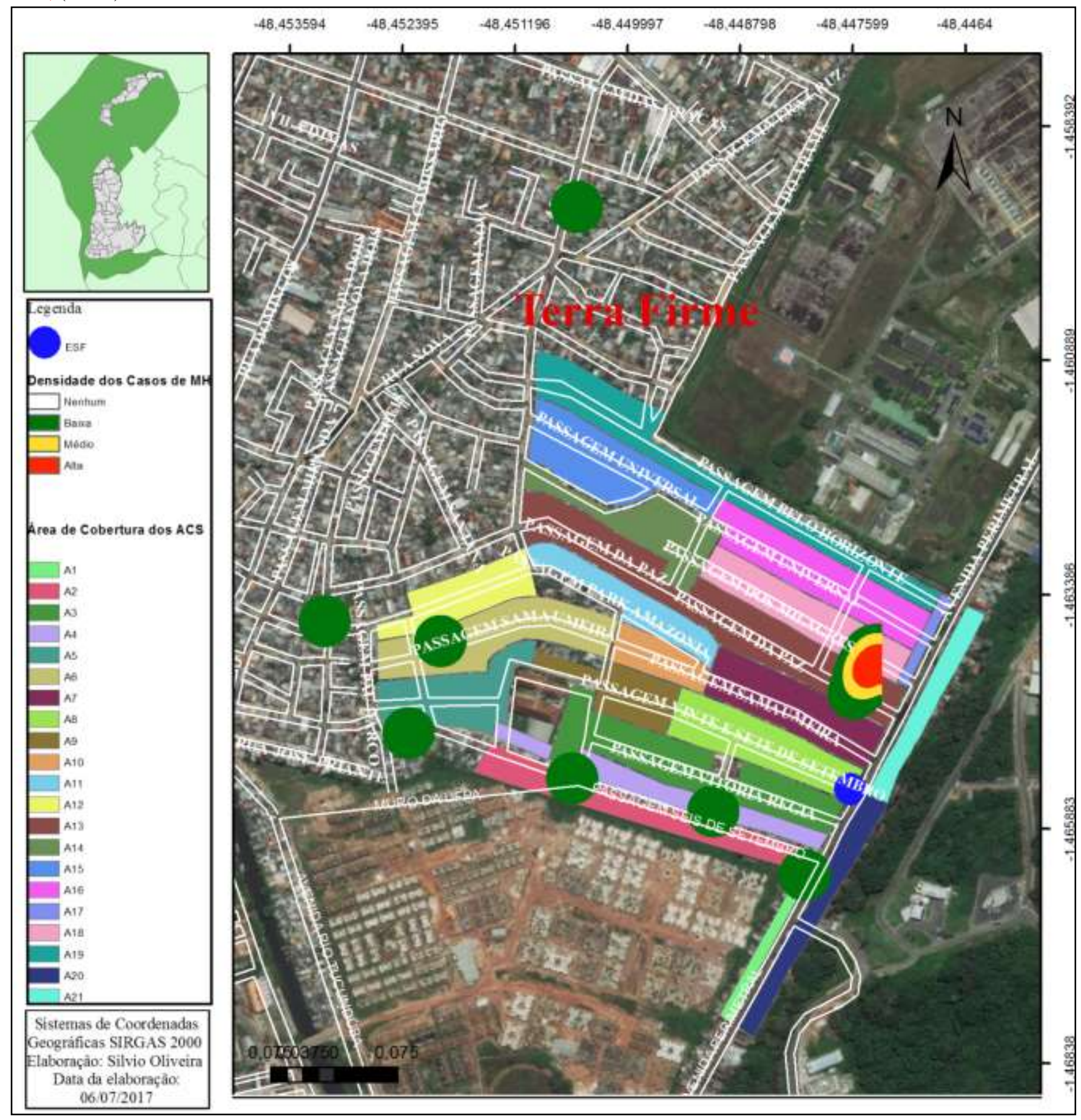

Fonte: Autores do trabalho (2017); Google maps, (2017); Qgis (2017).

\section{Discussão}

O predomínio de pacientes portadores de hanseníase do gênero masculino e cor da pele parda concorda com outras pesquisas (Melo, et al., 2014; Xavier, et al., 2014), além de representar a maioria da população brasileira - situação que pode 
estar relacionada com a miscigenação da população, especialmente na cidade de Belém (IBGE, 2010). A baixa escolaridade tem forte influência para a ocorrência de hanseníase, fato corroborado pela expressiva proporção de pacientes com ensino fundamental incompleto e de residentes em área de extrema pobreza observados neste estudo, concordando com outros anteriormente realizados (Macedo, et al., 2019; Schreuder, Noto \& Richardus, 2016; Duarte-Cunha, Cunha \& Souza-Santos, 2015).

A menor proporção de hanseníase encontrada em indivíduos menores de 15 anos pode estar relacionada com a falha na busca ativa de casos, quer no seio familiar quer entre escolares e se traduz em um resultado discordante de outras pesquisas realizadas (Schneider \& Freitas, 2016; Souza \& Rodrigues, 2015). É importante considerar, ainda, que a insuficiência de busca ativa entre os comunicantes de casos novos notificados e na população em geral, tem influência sobre o diagnóstico tardio, que pode acarretar o surgimento de incapacidades físicas (Ribeiro \& Lana, 2015). Nesse contexto, o maior adoecimento de indivíduos na faixa etária economicamente ativa corrobora o achado de outros estudos e traz prejuízos para a área econômica, devido o afastamento do paciente no início do tratamento ou ainda devido as formas incapacitantes presentes principalmente nos casos detectados tardiamente (Leano, et al., 2019; Costa, et al., 2017; Basso \& da Silva, 2017).

Cabe ressaltar que, embora correspondesse à menor porcentagem em relação à faixa etária, a população jovem pode estar relacionada à proporção de estudantes notificados como portadores de hanseníase. A maior frequência relativa de indivíduos apresentando a forma indeterminada, no ano de 2008, concorda estudos anteriores (Santos, 2015; Xavier, et al., 2014).

O registro da forma tuberculóide apenas no ano de 2010 difere de outras pesquisas no período (Monteiro, et al., 2017; Souza \& Rodrigues, 2015). A variabilidade de formas clínicas apresentada a partir de 2010 aduz uma mudança no padrão de acometimento dos indivíduos, onde a grande proporção da forma dimorfa em 2011, 2014 e 2015, passa a concordar com estudos anteriores (Macedo, et al., 2019; Souza \& Rodrigues, 2015). O grande número de casos notificados com as formas Virchowiana e dimorfa traz preocupação por constituir maior risco de alterações neurais irreversíveis e por traduzir-se em focos de transmissão ativa dentro da comunidade (Brasil, 2016).

A análise de padrões de distribuição de casos de hanseníase pelo método de Kernel permitiu visualizar onde ocorreu a maior concentração dos casos, evidenciando dessa forma diferentes densidades de interesse na superfície da área adstrita à ESF estudada. A identificação das duas microáreas onde se observa a maior concentração de residências de casos representa potencial interesse no estudo independentemente do cálculo de índices, porque oferece informações importantes para a execução de atividades de vigilância da saúde, mais especificamente no que se refere à busca ativa de casos, considerando a premissa atual da consolidação do modelo de atenção, que incorpora a territorialização no processo de trabalho visando cumprir os princípios constitucionais do SUS (Faria, 2020).

A percepção da dinâmica no atendimento da ESF, quando da aplicação da análise pelo método de Kernel identifica o atendimento de casos de hanseníase que residem fora da área adstrita dessa ESF, demonstrando a importância desse serviço para a integralidade da assistência, dado amparado por pesquisa anteriormente realizada (Brasil, 2017; Malta et al., 2016). Nesse contexto, em escala nacional, percebe-se que apesar do aumento do acesso ao sistema de saúde pela população a partir da implementação da ESF, ainda é necessário melhorar a cobertura destas nas áreas mais periféricas do município de forma que se possa oferecer cobertura universal e prioritária aos habitantes dessas localidades (Neves, et al., 2018). É importante atentar para as áreas de abrangência para atender à demanda de usuários, com redução do número de usuários vinculados às equipes, com o objetivo de evitar a sobrecarga aos profissionais e ao sistema e melhorar a qualidade do serviço ofertado (Tesser, Norman \& Vidal, 2018; Menezes, et al., 2017). 


\section{Conclusão}

A identificação do perfil clínico-epidemiológico associado aos casos de hanseníase no local de estudo possibilitou discutir, com a equipe da ESF, o direcionamento de planejamento à população com maior risco de adoecimento. Por meio de técnicas de análise espacial, a discriminação de áreas com densidade de casos notificados oferece informações importantes para o desenvolvimento prioritário das atividades de vigilância da saúde, que culminasse em busca ativa de casos, em um primeiro momento, nas duas microáreas identificadas, e expansão para as demais microáreas de cobertura da ESF. A territorialização dos casos permitiu identificar grande proporção de indivíduos residentes fora da área adstrita à ESF, tendo exposto a importância de utilização de técnicas de georreferenciamento na implementação de serviços necessários ao alcance da universalidade e equidade do SUS. Os resultados da pesquisa poderão servir de subsídio para a elaboração de um melhor planejamento e uma organização das atividades realizadas pela equipe de saúde da ESF em questão, bem como para as demais unidades que desenvolvem APS do município de Belém, assim como servir de base para novas pesquisas sobre o tema.

As limitações do estudo são inerentes à fonte dos dados, que depende do correto preenchimento pelos profissionais de saúde. Ressalta-se a necessidade de desenvolver estudos que busquem diagnosticar casos da doença nas demais ESF do município, incluindo a busca ativa em menores de 15 anos, visando comparar o perfil dos casos, bem como estimar a transmissão ativa da doença na capital.

\section{Referências}

Basso, M. E. M., \& da Silva, R. L. F. (2017). Perfil clínico-epidemiológico de pacientes acometidos pela hanseníase atendidos em uma unidade de referência. Revista da Sociedade Brasileira de Clínica Médica, 15(1), 27-32.

Benjak, A., Avanzi, C., Singh, P., Loiseau, C., Girma, S., Busso, P., \& Cole, S. T. (2018). Phylogenomics and antimicrobial resistance of the leprosy bacillus Mycobacterium leprae. Nature communications, 9(1), 1-11.

Brasil. Ministério da Saúde, Secretaria de Vigilância Epidemiológica em Saúde. (2014). Situação Epidemiológica da Hanseníase no Brasil. http://portalsaude.saude.gov.br/index.php?option=com_content \&view=article\&id=10995\&Itemid=661.

Brasil. Ministério da Saúde, Secretaria de Vigilância Epidemiológica em Saúde. (2017). Portaria $n^{\circ} 2.436$, de 21 de setembro de 2017. Aprova a Política Nacional de Atenção Básica, estabelecendo a revisão de diretrizes para a organização da Atenção Básica, no âmbito do Sistema Único de Saúde (SUS). http://www.brasilsus.com.br/index.php/legislacoes/gabinete-do-ministro/16247-portaria-n-2-436-de-21-de-setembro-de-2017

Brasil. Ministério da Saúde, Secretaria de Vigilância Epidemiológica em Saúde. (2016) Ministério da Saúde. Secretaria de Vigilância em Saúde. Departamento de Vigilância das Doenças Transmissíveis. Diretrizes para vigilância, atenção e eliminação da Hanseníase como problema de saúde pública: manual técnicooperacional file:///C:/Users/Evo/Downloads/831-Preprint\%20Text-1286-2-10-20200701.pdf

Costa, L. A., Borba-Pinheiro, C. J., dos Reis, J. H., \& dos Reis Júnior, S. H. (2017). Análise epidemiológica da hanseníase na Microrregião de Tucuruí, Amazônia brasileira, com alto percentual de incapacidade física e de casos entre jovens. Revista Pan-Amazônica de Saúde, 8(3), 8-8.

Duarte-Cunha, M., da Cunha, G. M., \& Souza-Santos, R. (2015). Geographical heterogeneity in the analysis of factors associated with leprosy in an endemic area of Brazil: are we eliminating the disease? BMC infectious diseases, 15(1), 1-8.

Faria, R. M. D. (2020). A territorialização da atenção básica à saúde do sistema único de saúde do Brasil. Ciência \& Saúde Coletiva, 25, 4521-4530.

IBGE. Instituto Brasileiro de Geografia e Estatística. (2010). Censo demográfico. http:/www.ibge.gov.br/home/estatística/censo2010a.

Leano, H. A. D. M., Araújo, K. M. D. F. A., Bueno, I. D. C., Niitsuma, E. N. A., \& Lana, F. C. F. (2019). Factores socioeconómicos relacionados con la lepra: revisión integrativa de la literatura. Revista Brasileira de Enfermagem, 72(5), 1405-1415.

Macedo G. M. M., Queiroz M. F. A., Silva A. R., Pinheiro B. V.S., Franco M. C. A., \& Xavier M. B. (2019). Distribuição espacial e evolução temporal da hanseníase em uma área de antiga colônia no estado do Pará. Revista Eletrônica Acervo Saúde, 11(12), e582

Malta, D. C., Santos, M. A. S., Stopa, S. R., Vieira, J. E. B., Melo, E. A., \& Reis, A. A. C. D. (2016). A cobertura da Estratégia Saúde da Família (ESF) no Brasil, segundo a Pesquisa Nacional de Saúde, 2013. Ciência \& Saúde Coletiva, 21, 327-338.

Melo, S. L., de Macedo, G. M. M., Pires, C. A. A., \& da Cunha, M. H. C. M. (2014). Recidiva hansênica em área de alta endemicidade no Estado do Pará, Brasil. Revista Pan-Amazônica de Saúde, 5(3), 6-6.

Menezes, E. L. C. D., Scherer, M. D. D. A., Verdi, M. I., \& Pires, D. P. D. (2017). Modos de produzir cuidado e a universalidade do acesso na atenção primária à saúde. Saúde e Sociedade, 26, 888-903. 
Research, Society and Development, v. 10, n. 4, e58110414375, 2021

(CC BY 4.0) | ISSN 2525-3409 | DOI: http://dx.doi.org/10.33448/rsd-v10i4.14375

Monteiro, M. J. D. S. D., dos Santos, G. M., Barreto, M. T. S., de Sousa Silva, R. V., de Jesus, R. L. R., \& da Silva, H. J. N. (2017). Perfil epidemiológico de casos de hanseníase em um estado do nordeste brasileiro. Revista de Atenção à Saúde, 15(54), 21-28.

Morosini, M. V. G. C., Fonseca, A. F., \& Lima, L. D. D. (2018). Política Nacional de Atenção Básica 2017: retrocessos e riscos para o Sistema Único de Saúde. Saúde em Debate, 42, 11-24.

Neves, R. G., Flores, T. R., Duro, S. M. S., Nunes, B. P., \& Tomasi, E. (2018). Tendência temporal da cobertura da Estratégia Saúde da Família no Brasil, regiões e Unidades da Federação, 2006-2016. Epidemiologia e Serviços de Saúde, 27, e2017170.

Pereira, A. S. et al. (2018). Metodologia da pesquisa científica. UFSM.

Ribeiro C. G. \& Lana, F. C. F. (2015). Incapacidades físicas em hanseníase: caracterização, fatores relacionados e evolução. Cogitare Enfermagem, 20(3), 496-503.

Santos, L. D. J. (2015). Caracterização espacial e temporal da endemia hansênica na zona urbana de Floriano-Piauí, 2004 a 2013.

Schreuder, P. A., Noto, S., \& Richardus, J. H. (2016). Epidemiologic trends of leprosy for the 21st century. Clinics in dermatology, 34(1), 24-31.

Schneider, P. B., \& Freitas, B. H. B. M. D. (2018). Tendência da hanseníase em menores de 15 anos no Brasil, 2001-2016. Cadernos de Saúde Pública, 34, e00101817.

Souza, C., \& Rodrigues, M. (2015). Magnitude, tendência e espacialização da hanseníase em menores de 15 anos no estado da bahia, com enfoque em áreas de risco: um estudo ecológico. Hygeia-Revista Brasileira de Geografia Médica e da Saúde, 11(20), 201-212.

Tesser, C. D., Norman, A. H., \& Vidal, T. B. (2018). Acesso ao cuidado na Atenção Primária à Saúde brasileira: situação, problemas e estratégias de superação. Saúde em Debate, 42, 361-378.

Xavier, M. B., Tavares, N. C. S., Corrêa, S. C.; Gonçalves, B. K.; Ramos, M. M. A. B., \& Macedo, G. M. M. (2014). Correlação Entre as Formas Clínicas da Hanseníase e o Grau de Incapacidade Neurológica. Revista Paraense de Medicina / Fundação Santa Casa de Misericórdia do Pará. - Belém: FSCMP, vol. $28(2)$.

WHO, World Health Organization (2015). Weekly Epidemiological Record. 36(90),461 - 476. http://www.who.int/wer. 